\title{
Asociación entre el polimorfismo rs9939609 del gen FTO y marcadores de adiposidad en población infantil chilena
}

\author{
Association between FTO gene rs9939609 and adiposity markers \\ in Chilean children
}

\author{
Natalia Ulloa ${ }^{\mathrm{a}, \mathrm{b}}$, Marcelo Villagrán ${ }^{\mathrm{c}}$, Benilde Riffo ${ }^{\mathrm{b}, \mathrm{c}}$, Andrea Gleisner ${ }^{\mathrm{d}}$, Fanny Petermann-Rocha ${ }^{\mathrm{e}}$, \\ Lorena Mardones ${ }^{\mathrm{c}}$, Ana María Leiva ${ }^{\mathrm{f}}$, María Adela Martínez-Sanguinetti ${ }^{\mathrm{g}}$, Carlos Celis-Morales ${ }^{\mathrm{h}, \mathrm{i}, \mathrm{j}}$ \\ (En representación del grupo ELHOC, Epidemiology of Lifestyle and Health Outcomes in Chile)
}

aCentro de Vida Saludable de la Universidad de Concepción. Concepción, Chile bDepartamento de Bioquímica Clínica e Inmunología, Facultad de Farmacia y de la Universidad de Concepción. Concepción, Chile ‘Departamento de Ciencias Básicas, Facultad de Medicina. Universidad Católica de la Santísima Concepción. Concepción. Chile 'Departamento de Pediatría, Facultad de Medicina. Universidad de Concepción. Concepción, Chile enstitute of Health and Wellbeing, University of Glasgow, Glasgow, United Kingdom fInstituto de Anatomía, Histología y Patología, Facultad de Medicina, Universidad Austral de Chile. Valdivia, Chile IInstituto de Farmacia, Facultad de Ciencias, Universidad Austral de Chile. Valdivia, Chile hBHF Glasgow Cardiovascular Research Centre, Institute of Cardiovascular and Medical Sciences, University of Glasgow. Glasgow, United Kingdom

'Centro de Investigación en Fisiología del Ejercicio (CIFE), Universidad Mayor. Santiago, Chile 'Laboratorio de Rendimiento Humano, Grupo de Estudio en Educación, Actividad Física y Salud (GEEAFyS), Universidad Católica del Maule. Talca, Chile

\section{¿Qué se sabe del tema que trata este estudio?}

La variante rs9939609 del gen FTO, ha sido ampliamenre reconocida a nivel mundial por su asociación con riesgo de obesidad en diversas poblaciones y edades, usando las clasificaciones de la OMS, basadas en IMC o límites de percentiles del IMC. Nuestro grupo también comunicó previamente que la variante rs9939609, del gen FTO, se asoció con un mayor riesgo de incremento del puntaje z del IMC en niños chilenos.

\section{¿Qué aporta este estudio a lo ya conocido?}

La obesidad se reconoce como un estado patológico que se caracteriza por un exceso de grasa en el cuerpo, específicamente en el tejido adiposo. En este estudio se evidencia la asociación entre la presencia del polimorfismo rs9939609 del gen FTO y el incremento de la adiposidad general (\% de masa grasa) y central (perímetro de cintura y razón cintura/talla), en población infantil de Chile.

Correspondencia:

Natalia Ulloa M

nulloa@udec.cl 


\section{Resumen}

La obesidad es una enfermedad inflamatoria donde la genética determina cierto nivel de riesgo. Aun cuando existen estudios que reportan asociación entre polimorfismos de FTO (fat-mass associated gene) y adiposidad, existe limitada evidencia en población infantil chilena. Objetivo: determinar la asociación entre el polimorfismo rs9939609 del FTO y marcadores de adiposidad en población infantil chilena. Pacientes y Método: Estudio de corte transversal incluyó 361 participantes (de 6 a 11 años; 50\% niñas). Los datos clínicos y la recolección de muestras de sangre se realizaron entre marzo y junio de 2008. El polimorfismo SNP (rs9939609), del gen FTO, se determinó utilizando ADN genómico extraído de leucocitos, utilizando el Mini Kit QIAamp DNA Blood (Qiagen GmbH, Hilden, Alemania). Los marcadores de adiposidad estudiados fueron, índice de masa corporal (IMC), masa grasa, perímetro de cintura (PC) y razón cintura/talla, y se compararon ajustados por sexo, edad y estadio de Tanner. La asociación entre el polimorfismo estudiado y los marcadores de obesidad se realizó mediante análisis de regresión lineal. Resultados: Al ajustar los marcadores por sexo, edad y estadío de Tanner se observó una asociación significativa entre el polimorfismo e indicadores de adiposidad. Por cada copia extra del alelo de riesgo se encontró un aumento de 2,47 kg de peso corporal, (IC 95\%: 1,39-3,55); 1,06 kg/m² de IMC, (IC 95\%: 0,56-1,54); $2,55 \mathrm{~cm}$ de PC, (IC 95\%: 1,26-3,85) y 1,98\% de masa grasa, (IC 95\%: 0,78-3,19). Al convertir los marcadores de adiposidad a $\mathrm{z}$-score, la razón perímetro de cintura/talla arrojó la mayor asociación con el alelo de riesgo de FTO. Conclusión: Este estudio indica asociación entre el polimorfismo rs9939609 del gen FTO con marcadores de adiposidad general y central en población infantil en Chile.

\section{Abstract}

Obesity is considered a chronic inflammatory disease with an important genetic component. Although several studies have reported an association between the FTO (fat-mass associated gene) and adiposity in children, there is limited evidence in the Chilean population. Objective: To determine the association between the polymorphism rs9939609 of the FTO gene and markers of adiposity in Chilean children. Patients and Method: Cross-sectional study which included 361 children aged between 6 and 11 years (50\% were girls). Between March and June 2008, clinical data and blood sample collection was carried out. The rs9939609 single-nucleotide polymorphism (SNP) of the FTO gene, was determined using the genomic DNA extracted from leukocytes, using the QIAamp DNA Blood Mini Kit (Qiagen GmbH, Hilden, Germany).The adiposity markers included were body mass index (BMI), waist circumference (WC), body fat, and WC/H index; which were later compared adjusted by sex, age, and Tanner stage. Linear regression analyses were conducted to detect the association between the polymorphism and obesity markers. Results: After adjusting the models by age, sex, and Tanner stage, we found a significant association between the polymorphism and markers of adiposity. For each extra copy of the risk allele, we found an increase of $2.47 \mathrm{~kg}$ body weight (95\% CI: 1.39-3.55); $1.06 \mathrm{~kg} / \mathrm{m}^{2}$ BMI (95\% CI: 0.56-1.54); $2.55 \mathrm{~cm} \mathrm{WC,} \mathrm{(95 \%} \mathrm{CI:} \mathrm{1.26-3.85);}$ and $1.98 \%$ body fat (95\% CI: $0.78-3.19)$. When converting adiposity markers to z-score, we found that WC/height index shows the strongest association with the risk allele FTO. Conclusion: This study supports the association between the rs9939609 SNP of the FTO gene and overall and central adiposity markers in Chilean children.
Palabras clave:

Obesidad;

FTO;

Genotipo;

Niños;

Índice de Masa

Corporal

Keywords:

Obesity;

FTO;

Genotype;

Children;

Body Mas Index

\section{Introducción}

La obesidad es una de las enfermedades que se puede prevenir, aún cuando en la actualidad es altamente prevalente afectando a más de 600 millones de personas en $2014^{1}$ y se asocia con la génesis y mayor prevalencia de enfermedades metabólicas crónicas ${ }^{7}$. Es por esto que la detención del incremento de los índices de obesidad constituye una de las nueve metas mundiales para prevenir y controlar las enfermedades crónicas no transmisibles que se ha propuesto la Organización Mundial de la Salud (OMS) para el año $2025^{8}$. Se ha estimado que el número de individuos obesos alcanzaría a 1,12 billones al $2030^{2,3}$.

En la población infantil, la obesidad es un problema de salud pública grave y urgente. Su prevalencia ha aumentado sustancialmente en niños y adolescentes, en países desarrollados y en países en vías desarrollo ${ }^{4}$. En Chile, el incremento de la obesidad infantil ha sido sostenido desde fines de la década de los años $80^{33,34}$. En 
la actualidad la prevalencia de exceso de peso corporal para los niveles de pre-kínder, kínder, primero básico y primero medio corresponden a 49,3\%, 50,8\%, 51,1\% y $44,5 \%$, respectivamente ${ }^{5,6}$. Este escenario corrobora que la obesidad infantil representa un problema de salud pública grave y urgente.

Entre los principales factores de riesgo modificables para el desarrollo de obesidad se encuentran la inactividad física, el sedentarismo, la magnitud de la ingesta energética y el patrón dietético tipo occidental ${ }^{9-11}$. Entre los potenciales factores de riesgo no modificables se encuentran el sexo, la edad, la etnia de origen, así como también los polimorfismos genéticos. Desde el año 2007, luego que se comunicara que el polimorfismo rs9939609 del gen FTO, presenta una asociación con índice de masa corporal (IMC) ${ }^{14}$, se ha incrementado rápidamente la identificación de polimorfismos genéticos de un solo nucleótido (SNP) asociados con incremento del IMC. En el año 2015, se habían identificado más de 97 polimorfismos genéticos asociados con mayor $\mathrm{IMC}^{12}$. Recientemente, un metaanálisis resumió la existencia de 738 SNP asociados con diversos marcadores de adiposidad (perímetro de cintura, masa grasa, tejido adiposo viceral, etc. $)^{13}$. De todas las variantes genéticas estudiadas, la variante rs9939609, del gen FTO (Fat-mass-associated-gene), es la que ha sido mayormente estudiada por su efecto reconocido en el aumento del IMC y riesgo de obesidad, confirmándose su asociación en distintas poblaciones de adultos y niños a nivel mundial ${ }^{14-18}$. Previamente reportamos que la variante rs9939609, del gen FTO, se asoció con un mayor riesgo de incremento del IMC en niños chilenos, pero no se evaluó su relación con otros marcadores de adiposidad ${ }^{18}$. El objetivo de este estudio fue investigar la asociación entre el polimorfismo rs9939609, del gen FTO, y marcadores de adiposidad general y central en esta misma población infantil chilena.

\section{Pacientes y Método}

Estudio de corte transversal en 361 niños de un entorno urbano de la Región del Biobío de Chile. Se incluyeron niños de un rango etario de 6 a 11 años de edad. Se excluyeron, aquellos niños que padecían cualquier patología crónica. Este estudio fue aprobado por el Comité de Bioética de la Universidad de Concepción. Los padres o tutores de los niños firmaron un consentimiento informado antes de la inclusión de su hijo en el estudio.

\section{Variables antropométricas: marcadores de adiposidad}

La talla se midió sin zapatos, utilizando estadiómetros montados en la pared con precisión de $0,1 \mathrm{~cm}$
(Seca, modelo 208). El peso corporal se midió con ropa ligera y sin zapatos en balanza Tanita (modelo TBF-300) con una precisión de $1 \mathrm{~g}$. El IMC $\left(\mathrm{kg} / \mathrm{m}^{2}\right)$ se calculó como el peso corporal dividido por la talla al cuadrado. La puntuación z del IMC, basada en la edad y el sexo, se calculó de acuerdo a las definiciones de la OMS. Los niños se clasificaron como normales (IMC $\geq 5 \mathrm{y}<85$ percentil) u obesos (IMC $>95$ percentil) de acuerdo con los percentiles internacionales de edad y género definidos por el Centro para el Control y Prevención de Enfermedades ${ }^{19}$. El perímetro de cintura (PC) se midió entre la costilla más baja y el borde superior de la cresta ilíaca, con una cinta métrica no distensible (Seca, modelo 201) con precisión de 0,1 $\mathrm{cm}$. La composición corporal se evaluó mediante análisis de impedancia bioeléctrica (TANITA, TBF-300). Todas las medidas fueron tomadas por nutricionistas entrenadas. Para definir el estadío puberal según los criterios de Tanner ${ }^{20}$, los niños fueron examinados por profesional médico.

\section{Determinación de variantes alélicas del gen FTO (Fat Mass Obesity-associated gene)}

Los datos clínicos y la recolección de muestras de sangre se realizaron entre marzo y junio de 2008. El polimorfismo SNP (rs9939609), del gen FTO, se determinó utilizando ADN genómico extraído de leucocitos, utilizando el Mini Kit QIAamp DNA Blood (Qiagen $\mathrm{GmbH}$, Hilden, Alemania), de acuerdo con el protocolo del fabricante. Las amplificaciones de la reacción en cadena de la polimerasa (PCR) se realizaron en un termociclador de PCR en tiempo real, Rotor-Gene 6500 (Corbett Research, Sydney, Australia), usando partidores previamente descritos por López-Bermejo et al. ${ }^{21}$ (directo: $5 \mathrm{~d}$ AACTG GCTCTTGAATGAAATAGGATTCAGA 3 'e inverso: 5' d AGAGTAACAGAGACTATCCAAGTGCATCAC 3'), bajo un protocolo previamente estandarizado ${ }^{18}$. La identificación del genotipo se realizó por comparación (Intervalos de confianza, IC 95\%) de los datos de fusión con genotipos patrones identificados mediante análisis de secuenciación en el Departamento de Ecología, Facultad de Ciencias Biológicas, Pontificia Universidad Católica de Chile. Para confirmar la existencia de un solo producto de PCR, se realizó una electroforesis con agarosa al 3\%. Todos los análisis de las muestras fueron realizados en duplicados, con un $98 \%$ de éxito en la determinación del genotipo.

\section{Análisis estadístico}

Los datos de caracterización de la población estudiada son presentados como promedio y desviación estándar (DE) para variables continuas, y como porcentaje para variables categóricas. Las diferencias entre genotipo fueron determinadas con análisis de re- 
gresión para variables continuas y con el test $\chi^{2}$ para variables categóricas.

Para investigar la asociación entre el gen FTO y los marcadores de obesidad: IMC, perímetro de cintura (PC), razón cintura/talla y \% de grasa corporal, se realizó análisis de regresión lineal. El genotipo del SNP rs9939609, del gen FTO, fue codificado siguiendo un modelo genético aditivo $(0=\mathrm{TT}$ - homocigoto para el alelo protector; $1=\mathrm{AT}$ - heterocigoto para el alelo de riesgo; 2 = AA - homocigoto para el alelo de riesgo), $y$ posteriormente, mediante análisis de regresión lineal se estimó el incremento en la variable de adiposidad por cada copia adicional de la variante de riesgo (alelo A). Estos resultados se presentan como promedio o coeficiente beta con sus respectivo intervalos de confianza del 95\% (IC 95\%).

Para determinar cuál de los marcadores de adiposidad presentó una mayor asociación con el gen FTO, todas las variables fueron estandarizadas a $\mathrm{z}$-score y así los resultados fueron presentados como coeficiente beta estandarizados y sus respectivo IC del $95 \%$, por cada copia adicional del alelo de riesgo del gen FTO.

Todos los análisis fueron ajustados por variables confundentes mediante la utilización de tres modelos estadísticos. Modelo 0 - sin ajustar; Modelo 1 - ajustado por edad y sexo; Modelo 2 - ajustado por el Modelo 1, pero también por estadío de Tanner. La distribución de equilibrio de Hardy-Weinberg de los alelos del gen FTO fue estimada mediante el test Chi-cuadrado en el programa STATA. Para todos los análisis se utilizó el programa STATA SE v14. El nivel de significancia fue definido como $\mathrm{p}<0,05$.

\section{Resultados}

La población estudiada se compone de 361 niños chilenos, en los cuales se midieron parámetros antropométricos y se genotipificó el SNP rs9939609 en el gen $F T O$, encontrando que la respectiva frecuencia alélica se distribuye siguiendo el equilibrio de Hardy-Weinberg (alelo $\mathrm{T}=0,649$ y alelo $\mathrm{A}=0,351$, $\left.\chi^{2}=0,053\right)$.

En la tabla 1 se presentan las características generales de la población, la cual correspondió a 178 niños y 183 niñas, de un rango de 6 a 11 años de edad (edad promedio: $8,51 \pm 1,44$ ). En un $79,8 \%$ de los niños se verificó un estadío de desarrollo en etapa prepuberal, según la clasificación de Tanner. El estado nutricional promedio arrojó un percentil $(87,0 \pm 16,6)$ con un porcentaje de $66,8 \%$ de niños y $65,0 \%$ de niñas obesas. La variante de riesgo en su forma heterocigota (genotipo TA) se expresó en un $27,9 \%$ de la población y en su forma homocigota (genotipo AA) en un $21,1 \%$ de la población.
Los resultados de la asociación entre SNP rs9939609 del gen $F T O$ y las variables de adiposidad son presentadas en la tabla 2 y figura 1. Estos resultados revelan que, en el modelo no ajustado por variables de confusión, todos los marcadores de adiposidad incrementan significativamente por cada copia extra del alelo de riesgo (A) del gen FTO. El incremento en los marcadores de adiposidad por cada copia extra del alelo de riesgo fue equivalente a: $2,19 \mathrm{~kg}$, (IC 95\%: 0,76; 3,61) para peso corporal; $2,28 \mathrm{~kg} / \mathrm{m}^{2}$ (IC 95\%: 0,47; 1,51) para IMC; $2,28 \mathrm{~cm}$, (IC 95\%: 0,81-0,75) para perímetro de cintura; y en $1,93 \%$, (IC 95\%: 0,69; 3,17) para \% de masa grasa. $\mathrm{Al}$ ajustar los modelos por las variables de confusión, modelo 1, edad y sexo y modelos 2 edad sexo y estadío de Tanner, la magnitud de la asociación entre el gen FTO y marcadores de adiposidad se mantuvo similar y en general aumentó el nivel de significancia estadística (tabla 2 y figura 1 ).

Tabla 1. Características generales de la población

\begin{tabular}{lc}
\hline Ancedentes generales & $\mathrm{n}(\%)$ \\
\hline N Total & $361(100)$ \\
Niñas, n (\%) & $183(50,7)$ \\
Estadío de Tanner prepuberal, n (\%) & $288(79,8)$ \\
Estadío de Tanner puberal, n (\%) & $73(20,2)$ \\
Normopeso, n (\%) & $123(34,1)$ \\
Obeso, n (\%) & $238(65,9)$ \\
\hline Variable & Promedio \pm SD \\
\hline Edad (años) & $8,5 \pm 1,4$ \\
Peso (kg) & $37,0 \pm 11,1$ \\
Talla (cm) & $131,5 \pm 10,1$ \\
Cintura (cm) & $72,3 \pm 11,4$ \\
IMC (kg/m $\left.{ }^{2}\right)$ & $21,5 \pm 4,0$ \\
IMC z score & $1,5 \pm 0,8$ \\
Percentil IMC & $87,0 \pm 16,6$ \\
Porcentaje grasa & $29,2 \pm 9,0$ \\
Masa grasa (kg) & $12,1 \pm 6,4$ \\
Masa magra (kg) & $26,9 \pm 5,4$ \\
Razón cintura/talla & $0,54 \pm 0,06$ \\
\hline Genotipo FTO (rs9939609) & $184(50,9)$ \\
\hline TT & $101(27,9)$ \\
\hline AA & $76(21,1)$ \\
\hline
\end{tabular}

Datos presentados como promedio y desviación estándar para variables de tipo continuas, y como número de participantes y su respectivo (\%) para variables de tipo categóricas. IMC: Índice de Masa Corporal. 
Tabla 2. Asociación entre el gen FTO (rs9939609) y marcadores de adiposidad

\begin{tabular}{|c|c|c|c|c|c|}
\hline \multirow[t]{2}{*}{ Variables } & \multicolumn{3}{|c|}{ Genotipo FTO rs9939609 (Promedio [IC 95\%]) } & \multirow{2}{*}{$\begin{array}{l}\text { Efecto del modelo genético } \\
\text { aditivo }\end{array}$} & \multirow[t]{2}{*}{ Valor $\mathrm{p}$} \\
\hline & $\mathrm{TT}$ & TA & AA & & \\
\hline \multicolumn{6}{|c|}{ Peso corporal (kg) } \\
\hline Modelo 0 & $36,6(35,0-38,2)$ & $38,0(35,8-40,13)$ & $41,2(38,8-43,7)$ & $2,19(0,76-3,61)$ & 0,003 \\
\hline Modelo 1 & $36,2(35,0-37,4)$ & $38,7(37,1-40,3)$ & $41,2(39,4-43,1)$ & $2,50(1,41-3,57)$ & $<0,0001$ \\
\hline Modelo 2 & $36,2(35,0-37,4)$ & $38,7(37,1-40,3)$ & $41,2(39,3-43,1)$ & $2,47(1,39-3,55)$ & $<0,0001$ \\
\hline \multicolumn{6}{|l|}{$I M C\left(k g / m^{2}\right)$} \\
\hline Modelo 0 & $20,9(20,3-21,4)$ & $21,8(20,0-22,5)$ & $22,9(21,0-23,9)$ & $0,99(0,47-1,51)$ & $<0,0001$ \\
\hline Modelo 1 & $20,8(20,3-21,3)$ & $21,9(21,2-22,6)$ & $22,9(22,1-23,7)$ & $1,06(0,57-1,54)$ & $<0,0001$ \\
\hline Modelo 2 & $20,8(21,2-21,3)$ & $21,9(21,2-22,6)$ & $22,9(22,0-23,7)$ & $1,06(0,56-1,54)$ & $<0,0001$ \\
\hline \multicolumn{6}{|c|}{ Perímetro de cintura $(\mathrm{cm})$} \\
\hline Modelo 0 & $70,8(69,2-72,4)$ & $72,6(70,4-74,8)$ & $75,5(73,0-78,1)$ & $2,28(0,81-3,75)$ & $<0,0001$ \\
\hline Modelo 1 & $70,5(69,0-71,9)$ & $73,2(71,2-75,1)$ & $75,6(73,3-77,8)$ & $2,56(1,27-3,85)$ & $<0,0001$ \\
\hline Modelo 2 & $70,5(69,0-71,9)$ & $73,2(71,2-75,1)$ & $75,6(73,3-77,8)$ & $2,55(1,26-3,85)$ & $<0,0001$ \\
\hline \multicolumn{6}{|c|}{ \% masa grasa } \\
\hline Modelo 0 & $28,1(26,7-29,4)$ & $29,2(27,4-31,0)$ & $32,2(30,0-34,4)$ & $1,93(0,69-3,17)$ & 0,002 \\
\hline Modelo 1 & $27,9(26,6-29,2)$ & $29,6(27,8-31,3)$ & $32,0(29,9-34,1)$ & $1,99(0,78-3,19)$ & 0,001 \\
\hline Modelo 2 & $27,9(26,6-29,3)$ & $29,6(27,8-31,4)$ & $32,0(29,9-34,1)$ & $1,98(0,78-3,19)$ & 0,001 \\
\hline
\end{tabular}

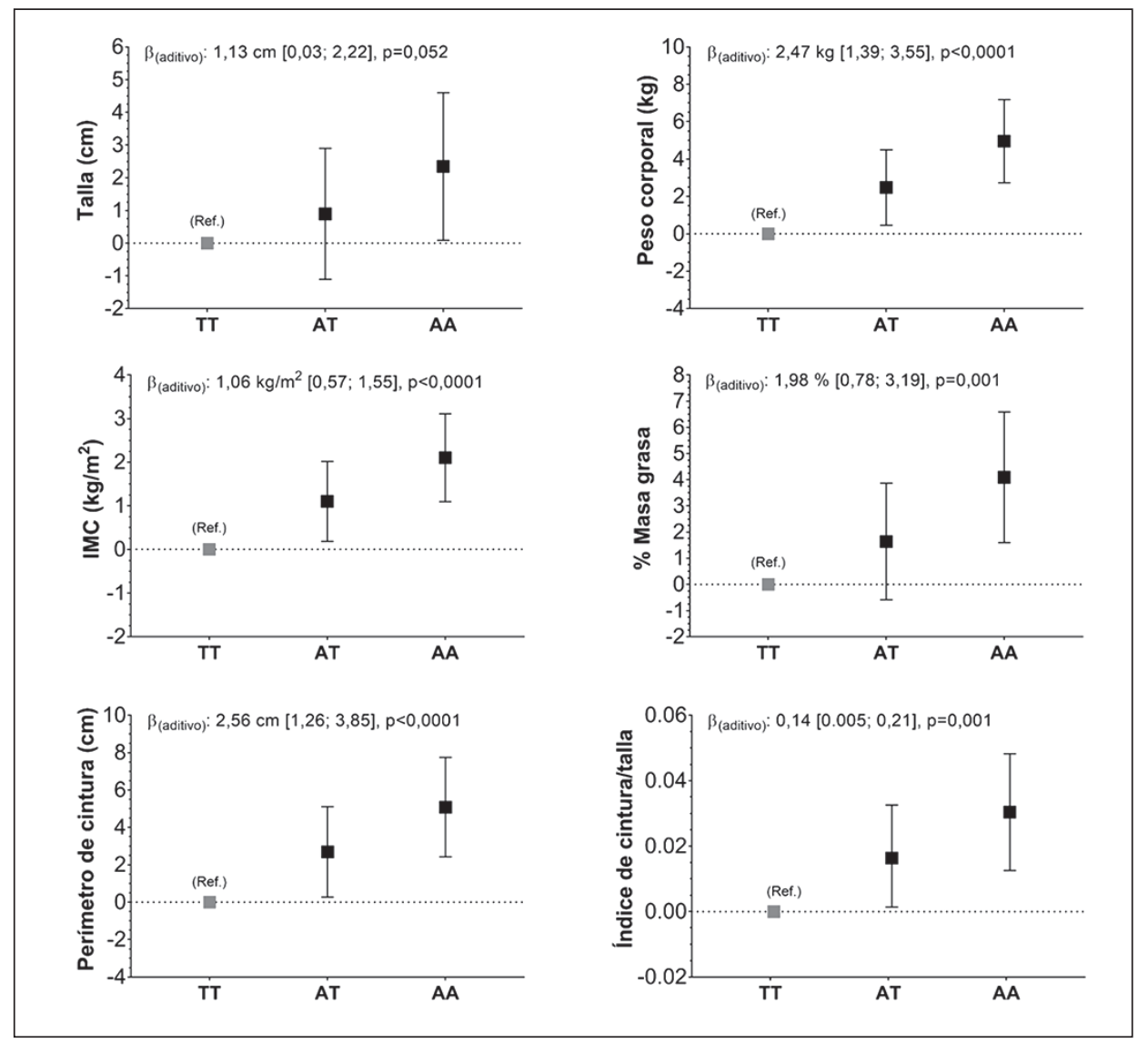

Figura 1. Asociación entre el gen FTO (rs9939609) y marcadores de adiposidad. Los resultados son presentados como promedio y su respectivo $95 \%$ de intervalo de confianza. El coeficiente beta aditivo representa el aumento en la variable de salida por cada copia extra del alelo de riesgo del gen FTO. LOS análisis fueron ajustados por edad, sexo y estadío de Tanner. 


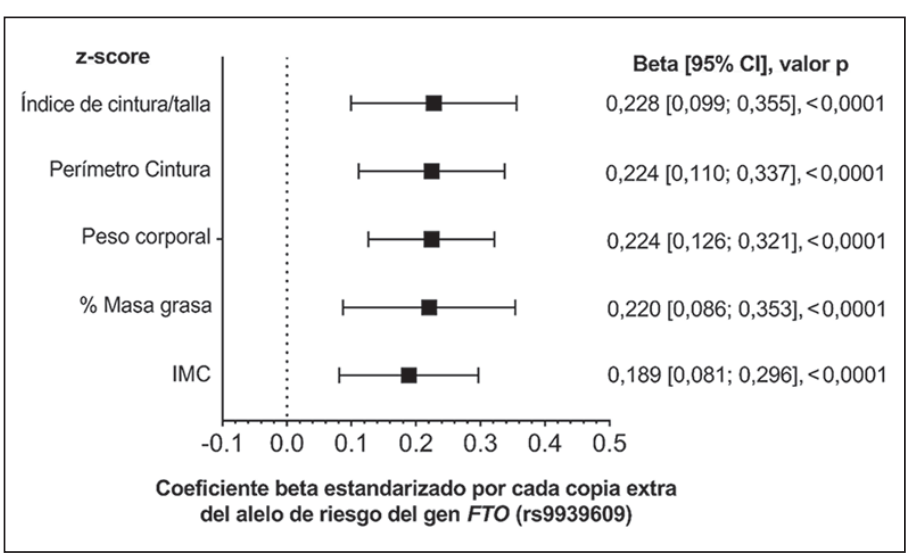

Figura 2. Asociación entre el gen FTO (rs9939609) y marcadores de adiposidad estandarizados a z-score. Datos son presentados como coeficiente beta estandarizados y sus respectivos intervalos de confianza del 95\% de intervalos de confianza. Este coeficiente representa el aumento en la variable de salida (variables de adiposidad) expresada como desviación estándar por cada copia extra del alelo de riesgo del gen FTO. Los análisis fueron ajustados por edad, sexo y estadío de Tanner.

Para comparar la fuerza de asociación de los marcadores de adiposidad con los genotipos de riesgo del gen FTO, estos fueron transformados a z-score, expresando la unidad de medida en términos de desviación estándar (DS) para cada uno de ellos. Estos resultados revelan que en el modelo estadístico más ajustado (modelo 2), los marcadores de adiposidad muestran la siguiente jerarquía de asociación con genotipos de riesgo en FTO (orden decreciente): (i) índice cintura/talla con 0,24 unidades de DS; (ii) perímetro de cintura con 0,22 unidades de DS; (iii) \% de masa grasa y finalmente; (iv) IMC con 0,19 unidades de DS (figura 2).

\section{Discusión}

El principal resultado de este estudio es que por cada copia extra del alelo de riesgo del rs9939609, del gen FTO, se observó una mayor probabilidad de incrementar la magnitud de los indicadores de adiposidad estudiados (\% de grasa corporal, perímetro de cintura, razón cintura/talla e IMCz).

Estos resultados indican que el polimorfismo rs9939609 del gen FTO contribuye a un mayor nivel de adiposidad en población infantil chilena.

Nuestro grupo de investigación había demostrado previamente que rs9939609 del gen FTO se asocia con un aumento del riesgo obesidad, expresado como razón de Odds ${ }^{18}$, en esta misma cohorte, compuesta por niños y niñas de 6 a 11 años de edad pertenecientes a una población urbana, de estrato social vulnerable de la región del Biobío de Chile. Sin embargo, en esta publicación no se examinó otros indicadores de adiposi- dad, ni se incluyó un análisis estadístico para atenuar la influencia de factores de confusión no modificables (edad, sexo, estadío de Tanner).

Diversos estudios han evidenciado que el FTO es un gen de susceptibilidad subyacente a la obesidad poligénica y que la influencia de FTO sobre el IMC cambia a lo largo de la vida en las poblaciones europeas ${ }^{22-24}$. En este estudio, la asociación entre el polimorfismo rs9939609 y los marcadores de adiposidad se mantuvo, a pesar de los posibles factores de confusión (edad, sexo o estadíos de Tanner). Se ha reportado que la edad de inicio de la asociación es desde los 7 años o incluso antes $^{25}$, lo cual es consistente con nuestros hallazgos de asociación de esta población infantil de 6 a 11 años de edad. Los niños de este estudio se encontraban mayoritariamente en un estadío prepuberal $(79,8 \%$ eran prepúberes), en tanto que estudios previos evidencian variación en rangos etarios más amplios, a lo largo de la primera y también de segunda década de vida ${ }^{23}$.

Por otro lado, previamente se ha reportado que las asociaciones del polimorfismo FTO con el IMC y la obesidad fueron más fuertes en las niñas que en los niños ${ }^{24,25}$, lo cual no se observó en este estudio. Estos resultados aparentemente contradictorios pueden deberse al bajo poder estadístico que resulta del reducido tamaño de muestra, junto con la relativa predominancia de un solo estadío de Tanner de niños y niñas (prepuberal). Es preciso indicar que tampoco se pueden excluir diferencias en la magnitud de exposición a ambientes obesogénicos de las poblaciones de los diferentes estudios. En este contexto, es fundamental la realización de nuevos estudios con un mayor tamaño muestra, que permita una mejor exploración de las posibles interacciones existentes entre edad, sexo y desarrollo puberal.

En población adulta chilena también se ha identificado asociación entre este polimorfismo del gen FTO y variables de adiposidad ${ }^{26}$, pero a diferencia del nuestro trabajo, no se identificó asociación con el perímetro de cintura. Lo anterior podría indicar que la distribución de la adiposidad cambia con la edad en los sujetos portadores del polimorfismo rs9939609 del gen FTO.

Esta observación es consistente con un estudio reciente, donde se encontró que la asociación de IMC con diversos loci de predisposición genética a obesidad cambia a lo largo del curso del desarrollo en los niños del Estudio Longitudinal de Santiago. Particularmente, se observó que la mayor asociación entre IMC-z score y la mayoría de los loci se producen a los 10 años de edad, a excepción de FTO, el que puede alcanzar su máximo efecto hasta los 16 años ${ }^{27}$.

Por otro lado, es importante establecer que una serie de autores han demostrado que los niños que portan la variable de riesgo FTO, presentan baja regulación del apetito y una mayor ingesta de alimen- 
$\operatorname{tos}^{28-31}$. Además, un metaanálisis mostró que la ingesta de proteínas en la dieta puede modificar la influencia de las variantes de FTO sobre el IMC ${ }^{32}$. Los hallazgos anteriores ofrecen una nueva visión de las interrelaciones entre las variantes genéticas de FTO, la ingesta de alimentos y la obesidad, pudiendo ser de utilidad para mejorar el diseño de programas de intervención para los niños, en el manejo de las conductas alimentarias y el aumento de peso excesivo.

Sin embargo, existen limitaciones que deben ser consideradas. Aunque el tamaño de la muestra otorga suficiente poder estadístico para detectar la asociación global de la variante genética rs9939609, del gen FTO, con indicadores de adiposidad; el tamaño de la muestra no fue suficiente para realizar análisis estratificados por edad, sexo o estadío puberal, entre el genotipo del gen FTO y los indicadores de adiposidad. Además, se analizó solamente la presencia del polimorfismo rs9939609 localizado en el primer intrón del gen FTO y hoy se sabe que el gen FTO es altamente polimórfico en toda su extensión, especialmente en intrones 1 y $8^{28}$. Nuestros análisis no nos permitieron descartar la presencia de otros SNPs del gen FTO en la población analizada. Tampoco se consignaron antecedentes sobre el estilo de vida de los niños (hábitos alimentarios, ejercicio físico y otros), los cuales podrían incidir en el resultado de asociación que reportamos entre el gen FTO y los marcadores de adiposidad.

En conclusión, el presente estudio indica la asociación del gen FTO y su polimorfismo rs9939609 con marcadores de adiposidad general y central en población infantil chilena y entrega evidencie que esta asociación es independiente de algunos factores de confusión no modificables (edad, sexo, estadío Tanner). Será de interés realizar futuros estudios que permitan evaluar factores de riesgo modificables, como la con- ducta alimentaria, el ejercicio físico y otros indicadores de estilo de vida de niños chilenos portadores de este polimorfismo del gen FTO y dilucidar cómo estas conductas o estilos de vida inciden en los marcadores de adiposidad en sujetos portadores de esta variante genética.

\section{Responsabilidades Éticas}

Protección de personas y animales: Los autores declaran que los procedimientos seguidos se conformaron a las normas éticas del comité de experimentación humana responsable y de acuerdo con la Asociación Médica Mundial y la Declaración de Helsinki.

Confidencialidad de los datos: Los autores declaran que han seguido los protocolos de su centro de trabajo sobre la publicación de datos de pacientes.

\section{Derecho a la privacidad y consentimiento informa-} do: Los autores han obtenido el consentimiento informado de los pacientes y/o sujetos referidos en el artículo. Este documento obra en poder del autor de correspondencia.

\section{Conflicto de intereses}

Los autores declaran no tener conflicto de intereses.

\section{Financiamiento}

\section{Proyecto INNOVA CORFO 07CN13ISM-19. Desarro-} llo de una estrategia territorial integrada transdisciplinaria para la prevención de la obesidad en la comunidad escolar.

\section{Referencias}

1. WHO 2016. Obesity and overweight. Disponible

en: http:// www.who.int/mediacentre/ factsheets/fs311/en/

2. Kelly T, Yang W, Chen C, et al. Global burden of obesity in 2005 and projections to 2030. Int J Obes. 2008;32:1431-7.

3. OECD. Obesity Update 2017. Organization for Economic Cooperation and Development. 2017. Disponible en: https://www.oecd.org/els/health.../ Obesity-Update-2017.pdf.

4. Ng M, Fleming T, Robinson M, Thomson B, el al. Global, regional, and national prevalence of overweight and obesity in children and adults during 1980-2013: a systematic analysis for the
Global Burden of Disease Study 2013. Lancet. 2014;384:766-81.

5. JUNAEB. Informe Mapa Nutricional 2015 [Internet]. JUNAEB; 2015 [último acceso: 8 de octubre de 2018]. Disponible en: https://www.junaeb.cl/wp-content/ uploads/2017/07/Informe-MapaNutricional-2015.pdf.

6. JUNAEB. Informe Mapa Nutricional 2016 [Internet]. JUNAEB 2017 [último acceso: 8 octubre de 2018]. Disponible en: http://www.ipsuss.cl/ipsuss/site/ artic/20170310/asocfile/20170310110609/ mapa_nutricional_2016_final.pdf.

7. Lloyd L, Langley-Evans S, McMullen S. Childhood obesity and risk of the adult metabolic syndrome: a systematic review. Int J Obes. 2012;36:1-11.

8. WHO. Marco mundial de vigilancia integral para la prevención y el control de las ENT. World Health Organization. 2011. Disponible en: http://www.who.int/ nmh/global_monitoring_framework/es/.

9. Alberti K, Eckel R, Grundy S, et al. Harmonizing the metabolic syndrome: a joint interim statement of the International Diabetes Federation Task Force on Epidemiology and Prevention; National Heart, Lung, and Blood Institute; American Heart Association; World Heart Federation; International Atherosclerosis Society; and International Association for the Study of Obesity. Circulation. 2009; 120:1640-5.

10. Dhalwani N, Zaccardi F, O’Donovan G, et al. Association Between Lifestyle Factors and the Incidence of Multimorbidity in an Older English Population. J Gerontol A Biol Sci Med Sci. 2017;72:528-34. 
11. Petermann F, Durán E, Labraña AM, et al. Risk factors for obesity: analysis of the 2009-2010 Chilean health survey. Rev Med Chile. 2017;145:716-22.

12. Locke A, Kahali B, Berndt S, et al. Genetic studies of body mass index yield new insights for obesity biology. Nature. 2015;518:197-206.

13. Dong S, Zhang Y, Chen Y, et al. Comprehensive review and annotation of susceptibility SNPs associated with obesity-related traits. Obesity Reviews. 2018;19:917-30.

14. Frayling T, Timpson N, Weedon M, et al. A common variant in the FTO gene is associated with body mass index and predisposes to childhood and adult obesity. Science. 2007;316:889-94.

15. González-Sánchez J, Zabena C, MartínezLarrad M, Martínez-Calatrava M, Pérez-Barba M, Serrano-Ríos M. Variant rs9939609 in the FTO gene is associated with obesity in an adult population from Spain. Clin Endocrinol. 2009;70:390-3.

16. Scuteri A, Sanna S, Chen W, et al. Genome-wide association scan shows genetic variants in the FTO gene are associated with obesity-related traits. PLoS Genet. 2007;3:e115.

17. Dina C, Meyre D, Gallina S, et al. Variation in FTO contributes to childhood obesity and severe adult obesity. Nat Genet. 2007;39:724-6.

18. Riffo B, Asenjo S, Sáez K, et al. FTO gene is related to obesity in Chilean Amerindian children and impairs HOMA-IR in prepubertal girls. Pediatric Diabetes. 2012;13:384-91.

19. CDC/NCHS CDC 2000 Growth Charts:
United States. http://www.chc.gov/ growthcharts.

20. Tanner JM, Whitehouse RH. Clinical longitudinal standards for height, weight, height velocity, weight velocity, and stages of puberty. Arch Dis Child. 1976;51:17079.

21. López-Bermejo A, Petry C, Díaz M, et al. The association between the FTO gene and fat mass in humans develops by the postnatal age of two weeks. J Clin Endocrinol Metab. 2008;93:1501-505.

22. Hardy R, Wills A, Wong A, et al. Life course variations in the associations between FTO and MC4R gene variants and body size. Hum Mol Genet. 2010;19:545-52.

23. Graff M, Ngwa JS, Workalemahu T, et al. Genome-wide analysis of BMI in adolescents and young adults reveals additional insight into the effects of genetic loci over the life course. Hum Mol Genet. 2013;22:3597-607.

24. Zhang M, Zhao X, Cheng H, et al. Age- and Sex-Dependent Association between FTO rs9939609 and ObesityRelated Traits in Chinese Children and Adolescents. PLoS ONE. 2014;9:e97545.

25. Hakanen M, Raitakari OT, Lehtimaki $\mathrm{T}$, et al. FTO genotype is associated with body mass index after the age of seven years but not with energy intake or leisure-time physical activity. J Clin Endocrinol Metab. 2009;94:1281-7.

26. Petermann F, Villagrán M, Troncoso C, et al. Association between FTO (ns9939609) genotype and adiposity markers in Chilean adults. Rev Med Chile. 2018;146:872-81.
27. Justice A, Chittoor G, Blanco E, et al. Genetic determinants of BMI from early childhood to adolescence: the Santiago Longitudinal Study. Pediatr Obes. 2019;14: e12479.

28. Cecil J, Tavendale R, Watt P, Hetherington M, Palmer C. An obesityassociated FTO gene variant and increased energy intake in children. $\mathrm{N}$ Engl J Med. 2008;359: 2558-66.

29. Wardle J, Llewellyn C, Sanderson S, Plomin R. The FTO gene and measured food intake in children. Int J Obesity. 2009;33:42-5.

30. Villagrán M, Petermann-Rocha F, Mardones L, et al. Association of the FTO (rs9939609) genotype with energy intake. Rev Med Chile. 2018;146:1252-60.

31. Emond J, Tovar A, Li Z, Lansigan R, Gilbert-Diamond D. FTO genotype and weight status among preadolescents: Assessing the mediating effects of obesogenic appetitive traits. Appetite. 2017;117:321-9.

32. Qibin, M. Downer, T. Kilpeläinen et al. Dietary Intake, FTO Genetic Variants, and Adiposity: A Combined Analysis of Over 16,000 Children and Adolescen. Diabetes. 2015;64:2467-476

33. Kain J, Uauy R, Lera L, Taibo M, Espejo F, Albala C. Evolution of the nutritional status of six years old Chilean children (1987-2003). Rev Med Chile. 2005;133:1013-20.

34. Herrera J, Lira M, Kain. Socioeconomic vulnerability and obesity in Chilean school children attending first grade: comparison between 2009 and 2013. Rev Chil Pediatr. 2017;88:736-43. 